TITLE:

\title{
Lepton flavor violation in the supersymmetric standard model with vectorlike leptons
}

AUTHOR(S):

Kitano, R; Yamamoto, K

CITATION:

Kitano, R ... [et al]. Lepton flavor violation in the supersymmetric standard model with vectorlike leptons. PHYSICAL REVIEW D 2000, 62(7): 073007.

\section{ISSUE DATE:}

2000-10-01

URL:

http://hdl.handle.net/2433/39893

RIGHT:

Copyright 2000 American Physical Society 
PHYSICAL REVIEW D, VOLUME 62, 073007

\title{
Lepton flavor violation in the supersymmetric standard model with vectorlike leptons
}

\author{
Ryuichiro Kitano \\ Theory Group, KEK, Oho 1-1, Tsukuba, Ibaraki 305-0801, Japan \\ and Department of Particle and Nuclear Physics, The Graduate University for Advanced Studies, \\ Oho 1-1, Tsukuba, Ibaraki 305-0801, Japan \\ Katsuji Yamamoto \\ Department of Nuclear Engineering, Kyoto University, Kyoto 606-8501, Japan
}

(Received 8 March 2000; published 6 September 2000)

\begin{abstract}
Lepton flavor violating processes are obtained from the mixing between ordinary leptons and vectorlike $\mathrm{SU}(2)_{L}$ doublet leptons which may originate in $E_{6}$. The effects of this lepton mixing are, however, suppressed naturally by the hierarchy of the charged lepton masses. In the supersymmetric model, significant effects of lepton flavor violation may appear rather through slepton mixing, which is in the present case generated by radiative corrections with ordinary-exotic lepton couplings. We are especially interested in the $\mu \rightarrow e \gamma$ decay. In the model without the bare mass term of vectorlike leptons, the supersymmetric contributions are rather suppressed due to the approximate $\mathrm{U}(1)_{e} \times \mathrm{U}(1)_{\mu}$. It is, however, remarkable that they are substantially enhanced by $\tan ^{6} \beta$. Then, $\operatorname{Br}(\mu \rightarrow e \gamma)$ might be comparable to the experimental bound for large $\tan \beta$. In the model with the bare mass term, much larger contributions are obtained through slepton mixing. These investigations show that the supersymmetric effects on lepton flavor violation due to the vectorlike leptons can be observed in the near future experiments.
\end{abstract}

PACS number(s): 14.60.Hi, 11.30.Fs, 12.60.Jv, 13.35.Bv

\section{INTRODUCTION}

The $E_{6}$ supersymmetric (SUSY) grand unified theory (GUT) and the $E_{8} \times E_{8}^{\prime}$ superstring theory are good candidates for unified theories of elementary particle physics. While such theories manifest themselves at the ultrahigh energy scale $\left(\geq 10^{16} \mathrm{GeV}\right)$, it is natural to expect that they have some effects on physics at the electroweak scale $\left(\sim 10^{2} \mathrm{GeV}\right)[1]$. It is, however, difficult to determine precisely what effective theory actually appears at the electroweak scale. This may be understood by considering the facts that the $E_{6}$ SUSY GUT has many possible symmetry breaking patterns and that the superstring theory has many possible compactification patterns. Hence, in the present experimental circumstances, it will not be so useful to perform detailed investigations on specific models. We are rather interested in the general aspects of the low-energy effects of the unified theories. Particularly, in this article we study the effects on lepton physics at the electroweak scale. In the standard model, the lepton number and flavor are conserved automatically. Hence, if the processes violating these quantum numbers are observed, including the neutrino masses and oscillations, $\mu \rightarrow e \gamma, \mu \rightarrow 3 e$, and so on, they indeed provide important information of new physics above the electroweak scale.

In the supersymmetric models with supergravity scenario [2], which would be derived from the unified theories, the renormalization effects on the soft supersymmetry breaking terms may induce sizable lepton flavor violation. These lepton flavor violating renormalization effects are actually obtained in the SUSY GUT [3-5] and the minimal supersymmetric standard model (MSSM) with heavy right-handed neutrinos [6-8]. Here, as another intriguing possibility for lepton flavor violation, we investigate the supersymmetric standard model incorporating vectorlike $\mathrm{SU}(2)_{L}$ doublet leptons $[9,10]$. Especially, the significant slepton mixing may be generated by radiative corrections with the couplings between the ordinary leptons and these exotic leptons. It is remarkable that this new physics with exotic leptons can be discovered just around the electroweak scale in contrast to the cases of GUT and right-handed neutrinos.

The present model is motivated by the $E_{6}$ type unification. The $E_{6} 27$ representation contains one generation of the ordinary quarks and leptons in the standard model, the vectorlike $\mathrm{SU}(2)_{L}$ singlet quarks, the vectorlike $\mathrm{SU}(2)_{L}$ doublet leptons, the right-handed neutrino, and the $\mathrm{SU}(2)_{L}$ singlet Higgs field. The ordinary left-handed neutrinos may acquire Majorana masses through the seesaw mechanism with the right-handed neutrinos [11]. These ordinary neutrino masses can be very small as required phenomenologically, if the right-handed neutrinos are superheavy or if they have extremely small Yukawa couplings with the left-handed lepton doublets. Hence, given the small ordinary neutrino masses, we do not consider further the effects of the right-handed neutrinos in the electroweak physics. In this investigation, we concentrate on the lepton flavor violating effects of the vectorlike lepton doublets $\left(L, L^{c}\right)$ and their scalar superpartners. Specifically, these supersymmetric effects of the exotic leptons may provide the significant $\operatorname{Br}(\mu \rightarrow e \gamma)$, while the lepton flavor changing neutral currents (FCNC's) at the tree level are suppressed naturally by the hierarchy of the charged lepton masses.

The down type quark singlets $\left(D^{c}, D\right) \sim \overline{\mathbf{3}}_{C}+\mathbf{3}_{C}$ are also present in the $E_{6}$ unification, which may form the $\overline{\mathbf{5}}+\mathbf{5}$ of $\mathrm{SU}(5)$ subgroup with $\left(L, L^{c}\right) \sim \mathbf{2}_{L}+\mathbf{2}_{L}$. The survival of these exotic particles to the electroweak scale depends on the symmetry breakings. The $\mathbf{2}_{L^{-}}-\mathbf{3}_{C}$ mass splitting in the $\mathbf{5}$ can be 
realized with suitable Higgs multiplets, e.g., the $\mathbf{3 5 1}$ of $E_{6}$ including the $\mathbf{2 4}$ of $\mathrm{SU}(5)$, as usually considered to obtain the light $\mathrm{SU}(2)_{L}$ doublet Higgs field. Hence, it is possible that $\left(L, L^{c}\right)$ survive while $\left(D^{c}, D\right)$ become superheavy in the 27. It may also be considered in the superstring theory that $\left(L, L^{c}\right)$ become light while $\left(D^{c}, D\right)$ do not in the $\overline{\mathbf{2 7}}+\mathbf{2 7}$. On the other hand, $\left(D^{c}, D\right)$ and $\left(L, L^{c}\right)$ are expected to survive together for the gauge coupling unification. Even in this case, $\left(D^{c}, D\right)$ and $\left(L, L^{c}\right)$ may originate in different 27's and/or $(\overline{\mathbf{2 7}}+\mathbf{2 7})$ 's. Then, it should be noted in these cases, whether $\left(D^{c}, D\right)$ survive or not, that the simple $E_{6}$ relations no longer hold for the Yukawa couplings after the $E_{6}$ breaking. In particular, the flavor violating couplings with $\left(L, L^{c}\right)$ may be described independently of those with $\left(D^{c}, D\right)$. Given this general situation, we concentrate on the lepton flavor violation with $\left(L, L^{c}\right)$ in the present article. A detailed examination of the quark flavor violation with $\left(D^{c}, D\right)$, particularly the supersymmetric effects, is reserved for a separate publication. (Such supersymmetric effects are investigated in Ref. [10] in a context different from the present one.) Of course, if $\left(D^{c}, D\right)$ and $\left(L, L^{c}\right)$ originate in the same 27 , the flavor violating effects of these exotic leptons and quarks will be provided from some common $E_{6}$ coupling. In this specific case, the calculations made in the present analysis for the lepton flavor violation such as $\mu \rightarrow e \gamma$ can be extended readily for the quark flavor violation such as $K^{0}-\bar{K}^{0}$ mixing. Simple order estimates, as will be quoted occasionally in the text, indicate that the constraints from the quark flavor violating effects are in fact less stringent than those from the lepton flavor violating effects.

This paper is organized as follows. In Sec. II, we describe the lepton mixing in the presence of vectorlike lepton doublets. Then, the contributions to $\operatorname{Br}(\mu \rightarrow e \gamma)$ due to the ordinary-exotic lepton mixing are shown to be negligibly small. In Sec. III, we investigate the slepton mixing generated by radiative corrections with the ordinary-exotic lepton couplings. Significant contributions to $\operatorname{Br}(\mu \rightarrow e \gamma)$ can be obtained from this slepton mixing. Sec. IV is devoted to the summary.

\section{THE LEPTON MIXING}

In this section, we describe the lepton mixing including the vectorlike lepton doublets, which contributes to the lepton flavor changing processes such as $\mu \rightarrow e \gamma$. We show that the modification of the gauge interactions provided by this lepton mixing is fairly suppressed even if the relevant flavor violating Yukawa couplings take significant values. This feature is indeed attributed to the mass hierarchy between ordinary leptons and exotic leptons.

The ordinary leptons are represented by $l_{i} \equiv\left(\nu_{i}, e_{i}\right)$ and $e_{i}^{c}$ with the subscript $i=1,2,3$ for the generations. The vectorlike leptons and singlet Higgs field are also listed as follows with their quantum numbers of the $\mathrm{SU}(2)_{L} \times \mathrm{U}(1)_{Y}$ :

$$
L \equiv l_{4}=\left(\begin{array}{l}
N \\
E
\end{array}\right):(\mathbf{2},-1 / 2), \quad L^{c}=\left(\begin{array}{c}
E^{c} \\
N^{c}
\end{array}\right):(\mathbf{2}, 1 / 2), \quad S:(\mathbf{1}, 0) .
$$

We here describe the case with one generation of $L$ and $L^{c}$ for simplicity. It is straightforward to extend the analysis for several generations of vectorlike leptons, and similar results are obtained for the lepton flavor violating processes. We examine two typical models in the following, for which distinct results will be obtained concerning the supersymmetric contributions to the lepton number violating processes such as $\mu \rightarrow e \gamma$.

In the first model, the superpotential relevant for the leptonic superfields is given by

$$
\mathcal{W}=\lambda_{1}^{a j} l_{a} H_{1} e_{j}^{c}+\lambda_{2}^{a 4} l_{a} L^{c} S+\lambda_{3} H_{1} H_{2} S+\frac{\lambda_{4}}{3} S^{3},
$$

where $a=i, 4$ and $i, j=1,2,3$. The Higgs fields include the pair of $\mathrm{SU}(2)_{L}$ doublets $H_{1}$ and $H_{2}$ and the singlet $S$. The vacuum expectation value (VEV) of $S$ gives the mass terms involving the exotic lepton doublets $L$ and $L^{c}$ as well as the $\mu$ term for the Higgs doublets $H_{1}$ and $H_{2}$. The $R$-parity invariance is assumed for the lepton number conservation. The $Z_{3}$ symmetry is also assumed to exclude the mass term of $S$ which is independent of the electroweak scale. (The cosmological domain wall problem associated with the spontaneous breaking of $Z_{3}$ may be evaded by introducing small explicit breaking in the higher order terms.) The coupling matrices in Eq. (2) may be simplified without loss of generality by redefinition of the relevant fields at some unification scale such as the gravitational scale $M_{G}$. The elements $\lambda_{1}^{4 j}$ in the $4 \times 3$ coupling matrix $\lambda_{1}$ can be eliminated by an appropriate unitary transformation among the left-handed doublets $l_{i}$ and $l_{4} \equiv L$. Then, the submatrix $\lambda_{1}^{i j}$ can be diagonalized by a redefinition of the ordinary leptons. Accordingly, we may take

$$
\lambda_{1}\left(M_{G}\right)=\left(\begin{array}{c}
\delta_{i j} \lambda_{1}^{i i} \\
\mathbf{0}
\end{array}\right)
$$

Furthermore, the components of $\lambda_{1}-\lambda_{4}$ can all be made real and non-negative.

In the second model, the above form of $\lambda_{1}$ is realized in terms of another $Z_{3}$, under which the nontrivial transformations of the relevant fields are given by

$$
L \rightarrow \omega L, \quad L^{c} \rightarrow \omega^{*} L^{c}, \quad S \rightarrow \omega S, H_{2} \rightarrow \omega^{*} H_{2}
$$

with $\omega^{3}=1$. Then, we have a bare mass term for $L$ and $L^{c}$ by making the following replacement in the superpotential (2):

$$
\lambda_{2}^{44} L L^{c} S[\operatorname{model}(1)] \rightarrow M_{L} L L^{c}[\operatorname{model}(2)] .
$$

In the lepton basis where the $\lambda_{1}\left(M_{G}\right)$ is given by Eq. (3), the ordinary-exotic lepton couplings $\lambda_{2}^{i 4}$ provide the source of lepton flavor violation. The lepton flavor violating elements in $\lambda_{1}$ are then generated at $M_{W}$ through the renormalization group evolution with these couplings $\lambda_{2}^{i 4}$. The renormalization group equations for the Yukawa couplings are given in the model (1) by 


$$
\begin{aligned}
\mu \frac{d}{d \mu} \lambda_{1}^{a j}= & \frac{1}{(4 \pi)^{2}}\left[3 \sum_{k=1}^{3} \sum_{b=1}^{4} \lambda_{1}^{a k} \lambda_{1}^{b k} \lambda_{1}^{b j}+\sum_{b=1}^{4} \lambda_{1}^{b j} \lambda_{2}^{a 4} \lambda_{2}^{b 4}\right. \\
& +\lambda_{1}^{a j}\left\{\sum_{b=1}^{4} \sum_{k=1}^{3}\left(\lambda_{1}^{b k}\right)^{2}+\lambda_{3}^{2}+3 \lambda_{4}^{2}\right\} \\
& \left.-\lambda_{1}^{a j}\left(3 g_{2}^{2}+3 g_{1}^{2}\right)\right] \\
\mu \frac{d}{d \mu} \lambda_{2}^{a 4}= & \frac{1}{(4 \pi)^{2}}\left[\lambda_{2}^{a 4}\left\{4 \sum_{b=1}^{4}\left(\lambda_{2}^{b 4}\right)^{2}+2 \lambda_{3}^{2}+2 \lambda_{4}^{2}\right\}\right. \\
& \left.+\sum_{b=1}^{4} \sum_{i=1}^{3} \lambda_{2}^{b 4} \lambda_{1}^{a i} \lambda_{1}^{b i}-\lambda_{2}^{a 4}\left(3 g_{2}^{2}+g_{1}^{2}\right)\right]
\end{aligned}
$$

The renormalization group equations in the model (2) are also obtained by setting $\lambda_{2}^{44}=0$ in the above equations. Then, we have the leading corrections for the off-diagonal elements of the $\lambda_{1}$ coupling as

$$
\lambda_{1}^{a j}\left(M_{W}\right)-\lambda_{1}^{a j}\left(M_{G}\right) \sim \frac{1}{(4 \pi)^{2}} \lambda_{1}^{j j} \lambda_{2}^{j 4} \lambda_{2}^{a 4} \log \frac{M_{G}}{M_{W}} \quad(a \neq j) .
$$

Hence, even if $\lambda_{1}^{a j}\left(M_{G}\right)=0 \quad(a \neq j)$ at $M_{G}$ as given in Eq. (3), these flavor changing elements of the $\lambda_{1}$ coupling arise at $M_{W}$. It should here be remarked that these corrections for $\lambda_{1}$ are in fact proportional to the corresponding diagonal elements $\lambda_{1}^{j j}$. It should also be mentioned that in the model (2) $\lambda_{1}^{4 j} \equiv 0$ and $\lambda_{2}^{44} \equiv 0$, which is ensured by the $Z_{3}$ symmetry (4).

The mass terms of the leptons are produced by the vacuum expectations values (VEV's) of the Higgs fields,

$$
\left\langle H_{1}\right\rangle=\left(\begin{array}{c}
v_{1} \\
0
\end{array}\right),\left\langle H_{2}\right\rangle=\left(\begin{array}{c}
0 \\
v_{2}
\end{array}\right),\langle S\rangle=v_{3},
$$

where $v=\left(v_{1}^{2}+v_{2}^{2}\right)^{1 / 2} \simeq 174 \mathrm{GeV}$ and $\tan \beta=v_{2} / v_{1}$. These VEV's may acquire nonvanishing phases introducing a new source of $C P$ violation. We, however, do not consider this possibility for simplicity, and therefore take the real VEV's. Then, the charged lepton mass matrix is given at $M_{W}$ by

$$
M_{\mathcal{E}}=\left(\begin{array}{cc}
\lambda_{1}^{i j} v_{1} & \lambda_{2}^{i 4} v_{3} \\
\lambda_{1}^{4 j} v_{1} & \lambda_{2}^{44} v_{3}
\end{array}\right)
$$

It should here be remembered that $\lambda_{2}^{44} v_{3} \rightarrow M_{L}$ in the model (2). This $4 \times 4$ real matrix is diagonalized by two orthogonal matrices $U_{\mathcal{E}}$ and $V_{\mathcal{E}}$ as

$$
U_{\mathcal{E}}^{T} M_{\mathcal{E}} V_{\mathcal{E}}=\operatorname{diag}\left(m_{e}, m_{\mu}, m_{\tau}, M_{E}\right) .
$$

The neutral lepton mass matrix is also produced as

$$
M_{\mathcal{N}}=\left(\begin{array}{c}
-\lambda_{2}^{i 4} v_{3} \\
-\lambda_{2}^{44} v_{3}
\end{array}\right)
$$

This $4 \times 1$ real matrix is diagonalized with an orthogonal matrix $O_{\mathcal{N}}$ as

$$
O_{\mathcal{N}}^{T} M_{\mathcal{N}}=\left(\begin{array}{c}
0 \\
M_{N}
\end{array}\right)
$$

The masses of the ordinary charged leptons are given by

$$
m_{e_{i}} \simeq \lambda_{1}^{i i} v_{1}
$$

where the radiative corrections (8) for the $\lambda_{1}$ coupling are considered. The masses of the exotic leptons are also given by

$$
M_{E} \simeq M_{N}=v_{3}\left[\sum_{a=1}^{4}\left(\lambda_{2}^{a 4}\right)^{2}\right]^{1 / 2} .
$$

The zero modes in Eq. (13) are the three generations of ordinary neutrinos. It is noticed that the orthogonal matrix $O_{\mathcal{N}}$ is not determined uniquely at this stage. An arbitrary $3 \times 3$ transformation, say $O_{\nu}^{\prime}$, to redefine the (approximately) massless ordinary neutrinos can be incorporated in $O_{\mathcal{N}}$. The ordinary neutrinos may acquire very small Majorana masses through the seesaw mechanism with the right-handed neutrinos of $\mathrm{SU}(2)_{L}$ singlet. Then, the neutrino mixing matrix $O_{\mathcal{N}}$ is determined completely.

We here consider some relevant properties of the transformation matrices $U_{\mathcal{E}}, V_{\mathcal{E}}$, and $O_{\mathcal{N}}$. First, the orthogonal transformation $V_{\mathcal{E}}$, which represents the right-handed charged lepton mixing, diagonalizes the matrix $M_{\mathcal{E}}^{T} M_{\mathcal{E}}$. By considering the structure of $M_{\mathcal{E}}^{T} M_{\mathcal{E}}$ from Eq. (10) with Eq. (8), the right-handed ordinary-exotic lepton mixing in $V_{\mathcal{E}}$, which is relevant for describing the gauge interactions as seen below, is estimated in the leading order (with $M_{E} \sim v_{3}$ for definiteness) as

$$
\left(V_{\mathcal{E}}\right)_{i 4} \simeq-\left(V_{\mathcal{E}}\right)_{4 i} \sim\left(m_{e_{i}} / M_{E}\right) \lambda_{2}^{i 4} \text {. }
$$

It should here be remarked that the right-handed lepton mixing effects are suppressed sufficiently by the mass ratios $m_{e_{i}} / M_{E}$ with the experimental bound $M_{E}>97.0 \mathrm{GeV}$ [12], even if the ordinary-exotic lepton couplings $\lambda_{2}^{i 4}$ take significant values. On the other hand, the left-handed ordinaryexotic lepton mixings in $U_{\mathcal{E}}$ and $O_{\mathcal{N}}$ are estimated as

$$
\left(U_{\mathcal{E}}\right)_{i 4} \simeq-\left(U_{\mathcal{E}}\right)_{4 i} \simeq\left(O_{\mathcal{N}}\right)_{i 4} \simeq-\left(O_{\mathcal{N}}\right)_{4 j}\left(O_{\nu}^{\prime}\right)_{j i} \sim \lambda_{2}^{i 4},
$$

where the ambiguity in $O_{\mathcal{N}}$ as mentioned above is extracted explicitly by suitably choosing the orthogonal transformation $O_{\nu}^{\prime}$ of the ordinary neutrinos. These left-handed mixings are no longer suppressed by $m_{e_{i}} / M_{E}$. Here, we note the similarity between $U_{\mathcal{E}}$ for the charged leptons and $O_{\mathcal{N}}$ for the neutral leptons. In fact, the effective combination of lefthanded lepton mixing is estimated as

$$
\left(U_{\mathcal{E}}^{T} O_{\mathcal{N}}\right)_{i 4} \simeq-\left(U_{\mathcal{E}}^{T} O_{\mathcal{N}}\right)_{4 j}\left(O_{\nu}^{\prime}\right)_{j i} \sim\left(m_{e_{i}} / M_{E}\right)^{2} \lambda_{2}^{i 4},
$$




$$
\left(U_{\mathcal{E}}^{T} O_{\mathcal{N}}\right)_{i k}\left(O_{\nu}^{\prime}\right)_{k j}-\delta_{i j} \sim\left(m_{e_{i}} / M_{E}\right)\left(m_{e_{j}} / M_{E}\right) \lambda_{2}^{i 4} \lambda_{2}^{j 4}
$$

This similarity of the left-handed lepton mixings described in terms of the $\lambda_{2}^{i 4}$ couplings and the ordinary-exotic lepton mass ratios is indeed stable against the radiative corrections. It can be understood by noting the following facts. (i) By setting $\lambda_{1}^{i i}=0$ at $M_{G}$ in Eq. (8), we have $\lambda_{1}^{a j} \equiv 0$ at any scales as seen from Eq. (6). Then, $M_{\mathcal{E}}$ essentially coincides with $M_{\mathcal{N}}$, and they are diagonalized with the left-handed lepton transformation $U_{\mathcal{E}}=O_{\mathcal{N}}$ to eliminate the $\lambda_{2}^{i 4} v_{3}$ term. (ii) The change $\lambda_{1} \rightarrow-\lambda_{1}$ of the Yukawa coupling in $M_{\mathcal{E}}$ is compensated by the change $e_{i}^{c} \rightarrow-e_{i}^{c}$ of the right-handed ordinary leptons. This means that the sign change of $\lambda_{1}$ does not affect the left-handed lepton mixing. Hence, by considering (i) and (ii) we find that the difference between $U_{\mathcal{E}}$ and $O_{\mathcal{N}}$ should appear at the second order of $\lambda_{1}^{a j}\left(M_{W}\right) \sim \lambda_{1}^{j j}$ $\sim m_{e_{j}} / v_{1}$. (iii) By setting $\lambda_{2}^{i 4}\left(M_{G}\right)=0$ so that $\lambda_{2}^{i 4}=0$ at any scales, the $\lambda_{1}$ coupling remains to be diagonal even if the radiative corrections are included. Hence, in this limit with $\lambda_{2}^{i 4}=0, M_{\mathcal{E}}$ and $M_{\mathcal{N}}$ are diagonal, and the complete similarity follows with $U_{\mathcal{E}}=O_{\mathcal{N}}=\mathbf{1}_{4}$ ( $4 \times 4$ unit matrix).

It can be shown that the flavor violating gauge couplings of leptons are suppressed naturally due to the smallness of $\left(V_{\mathcal{E}}\right)_{i 4}$ and the similarity between $U_{\mathcal{E}}$ and $O_{\mathcal{N}}$. The charged gauge interaction coupled to $W$ boson is given by

$$
\begin{aligned}
\mathcal{L}_{W}= & -\frac{g_{2}}{\sqrt{2}}\left(U_{\mathcal{E}}^{T} O_{\mathcal{N}}\right)_{a b} \overline{\mathcal{E}}_{a} \gamma^{\mu} P_{L} \mathcal{N}_{b} W_{\mu}^{-} \\
& +\frac{g_{2}}{\sqrt{2}}\left(V_{\mathcal{E}}\right)_{4 a} \overline{\mathcal{E}}_{a} \gamma^{\mu} P_{R} \mathcal{N}_{4} W_{\mu}^{-}+\text {H.c. }
\end{aligned}
$$

where $\mathcal{E}_{a} \equiv\left(e_{i}, E\right)$ and $\mathcal{N}_{a} \equiv\left(\nu_{i}, N\right)(a=1-4)$ represent the four-component Dirac spinors of the lepton mass eigenstates, and $P_{L}$ and $P_{R}$ are the chiral projections to the left-handed and right-handed parts, respectively. This charged gauge interaction is relevant for the $\mu \rightarrow e \gamma$ decay. The lepton flavor violating terms with the factors $\left(U_{\mathcal{E}}^{T} O_{\mathcal{N}}\right)_{a b}(a \neq b)$ are apparently induced in the left-handed couplings of Eq. (20). However, due to the similarity between $U_{\mathcal{E}}$ and $O_{\mathcal{N}}$ as shown in Eqs. (18) and (19), they are almost rotated out with the suitable redefinition $O_{\nu}^{\prime}$ of the ordinary neutrinos neglecting the tiny neutrino masses $m_{\nu_{i}}$. The flavor changing right-handed couplings are also suppressed by the small factors $\left(V_{\mathcal{E}}\right)_{4 i}$, as shown in Eq. (16). Hence, the effects of the charged gauge interaction appear to be extremely small for the lepton flavor violating processes.

The neutral gauge interaction of charged leptons coupled to $Z$ boson is given by

$$
\begin{aligned}
\mathcal{L}_{Z}= & \mathcal{L}_{Z}^{0}+\frac{1}{2}\left(g_{2} \cos \theta_{W}+g_{1} \sin \theta_{W}\right) \\
& \times\left(V_{\mathcal{E}}\right)_{4 a}\left(V_{\mathcal{E}}\right)_{4 b} \overline{\mathcal{E}}_{a} \gamma^{\mu} P_{R} \mathcal{E}_{b} Z_{\mu},
\end{aligned}
$$

where $\mathcal{L}_{Z}^{0}$ represents the usual flavor diagonal part which is obtained by turning off the ordinary-exotic lepton mixing. The lepton flavor violating term appears only in the righthanded couplings of Eq. (21). This is because the left-handed leptons all form the $\mathrm{SU}(2)_{L}$ doublets. Since the FCNC's of ordinary leptons in Eq. (21) are proportional to $\left(V_{\mathcal{E}}\right)_{4 i}\left(V_{\mathcal{E}}\right)_{4 j}$, their effects are fairly suppressed by the small mass ratios $\left(m_{e_{i}} / M_{E}\right)\left(m_{e_{j}} / M_{E}\right)$. If the quark singlets $\left(D^{c}, D\right)$ are also present, they may have the flavor violating coupling which has the common $E_{6}$ origin with the $\lambda_{2}$ coupling of $\left(L, L^{c}\right)$. In this case, the FCNC's of ordinary quarks appear as well to be negligibly small due to the suppression factors $\left(m_{d_{i}} / M_{D}\right)\left(m_{d_{j}} / M_{D}\right)$.

We now estimate the contributions to the $\mu \rightarrow e \gamma$ decay which are provided by these gauge interactions. We will in fact observe below that these contributions are negligibly small. The decay amplitude of $\mu \rightarrow e \gamma$ is generally given by

$T(\mu \rightarrow e \gamma)=e \epsilon^{\alpha *} \bar{u}_{e}(p-q)\left[i \sigma_{\alpha \beta} q^{\beta}\left(A_{L} P_{L}+A_{R} P_{R}\right)\right] u_{\mu}(p)$.

Then, the decay rate is calculated by

$$
\Gamma(\mu \rightarrow e \gamma)=\frac{1}{16 \pi} e^{2} m_{\mu}^{3}\left(\left|A_{L}\right|^{2}+\left|A_{R}\right|^{2}\right) .
$$

The contributions to the amplitudes $A_{L}$ and $A_{R}$ are provided by the one-loop diagrams with the intermediate states of the neural leptons ( $W$ mediated) and charged leptons ( $Z$ mediated). We can see from Eqs. (16)-(19) that all these diagrams include the significant suppression factor $\left(m_{e} /\right.$ $\left.M_{E}\right)\left(m_{\mu} / M_{E}\right) \sim 10^{-8}$. Then, the $W$ and $Z$ contributions to the decay amplitude are given by

$$
A_{L, R}^{(W)} \sim A_{L, R}^{(Z)} \sim \frac{1}{32 \pi^{2}} \frac{m_{e} m_{\mu}^{2}}{M_{W}^{2} M_{E}^{2}} \lambda_{2}^{14} \lambda_{2}^{24} .
$$

Therefore, the flavor violating gauge couplings induced by the ordinary-exotic lepton mixing provide tiny contributions $\sim 10^{-21}$ to the branching ratio $\operatorname{Br}(\mu \rightarrow e \gamma)$. This feature is actually confirmed by numerical calculations.

\section{THE SLEPTON MIXING}

In the supersymmetric model, significant effects of flavor violation may be obtained through the slepton mixing. It is expected that the supersymmetry breaking is provided in the minimal supergravity model with the soft terms at the gravitational scale $M_{G} \sim 10^{18} \mathrm{GeV}$, including the common slepton masses squared $m_{\tilde{l} a b}^{2}=m_{0}^{2} \delta_{a b}$, etc., the $A$ terms $A_{k}$ $=a_{0} m_{0} \lambda_{k}$, and the gaugino masses $m_{1}$ and $m_{2}$. We have seen that in the present sort of models the original source of lepton flavor violation is the ordinary-exotic lepton couplings $\lambda_{2}^{i 4}$. At the tree-level with the universal soft supersymmetry breaking terms, however, the flavor violating effects of these couplings are still suppressed by the ordinary-exotic lepton mass ratio $m_{e_{i}} / M_{E}$, since the lepton and slepton mass matrices are diagonalized simultaneously in this limit. Significant effects of flavor violation are rather provided from the 
radiative corrections on the soft terms which are generated with the ordinary-exotic lepton couplings $\lambda_{2}^{i 4}$.

The soft mass terms and $A$-terms at the electroweak scale $M_{W}$ deviate from the universal forms through the renormalization group evolution [13]. In the leading order approximation the corrections are given for the model (1) by

$$
\begin{aligned}
& \Delta m_{\tilde{l}_{a a}}^{2}=-\frac{1}{(4 \pi)^{2}}\left[2 m_{0}^{2}\left(3+a_{0}^{2}\right)\left\{\left(\lambda_{1}^{a a}\right)^{2}+\left(\lambda_{2}^{a 4}\right)^{2}\right\}\right. \\
& \left.-\left(6 m_{2}^{2} g_{2}^{2}+2 m_{1}^{2} g_{1}^{2}\right)\right] \log \frac{M_{G}}{M_{W}}, \\
& \Delta m_{\tilde{l}_{a b}}^{2}=-\frac{2}{(4 \pi)^{2}} m_{0}^{2}\left(3+a_{0}^{2}\right) \lambda_{2}^{a 4} \lambda_{2}^{b 4} \log \frac{M_{G}}{M_{W}} \quad(a \neq b), \\
& \Delta m_{\tilde{e}^{c} i j}^{2}=-\frac{\delta_{i j}}{(4 \pi)^{2}}\left[4 m_{0}^{2}\left(\lambda_{1}^{i i}\right)^{2}\left(3+a_{0}^{2}\right)-8 g_{1}^{2} m_{1}^{2}\right] \log \frac{M_{G}}{M_{W}}, \\
& \Delta m_{\tilde{L} c}^{2}=-\frac{1}{(4 \pi)^{2}}\left[2 m_{0}^{2}\left(3+a_{0}^{2}\right) \sum_{a=1}^{4}\left(\lambda_{2}^{a 4}\right)^{2}\right. \\
& \left.-\left(6 g_{2}^{2} m_{2}^{2}+2 g_{1}^{2} m_{1}^{2}\right)\right] \log \frac{M_{G}}{M_{W}} \\
& \Delta A_{1}^{i i}=-\frac{1}{(4 \pi)^{2}} \lambda_{1}^{i i}\left[3 a _ { 0 } m _ { 0 } \left\{\left(\lambda_{2}^{i 4}\right)^{2}+\lambda_{3}^{2}+3 \lambda_{4}^{2}+3\left(\lambda_{1}^{i i}\right)^{2}\right.\right. \\
& \left.+\sum_{j=1}^{3}\left(\lambda_{1}^{j j}\right)^{2}\right\}-3 a_{0} m_{0}\left(g_{2}^{2}+g_{1}^{2}\right) \\
& \left.-6\left(g_{2}^{2} m_{2}+g_{1}^{2} m_{1}\right)\right] \log \frac{M_{G}}{M_{W}}, \\
& \Delta A_{1}^{a j}=-\frac{3}{(4 \pi)^{2}} a_{0} m_{0} \lambda_{1}^{j j} \lambda_{2}^{a 4} \lambda_{2}^{j 4} \log \frac{M_{G}}{M_{W}} \quad(a \neq j), \\
& \Delta A_{2}^{a 4}=-\frac{1}{(4 \pi)^{2}} \lambda_{2}^{a 4}\left[3 a _ { 0 } m _ { 0 } \left\{4 \sum_{b=1}^{4}\left(\lambda_{2}^{b 4}\right)^{2}+2 \lambda_{3}^{2}+2 \lambda_{4}^{2}\right.\right. \\
& \left.+\left(\lambda_{1}^{a a}\right)^{2}\right\}-a_{0} m_{0}\left(3 g_{2}^{2}+g_{1}^{2}\right) \\
& \left.-2\left(3 g_{2}^{2} m_{2}+g_{1}^{2} m_{1}\right)\right] \log \frac{M_{G}}{M_{W}} .
\end{aligned}
$$

We should set $\lambda_{2}^{44}=0$ for the model (2) in these formulas. Because of these radiative corrections, the slepton mass matrices and the corresponding lepton mass matrices are no longer diagonalized simultaneously, providing the new source of lepton flavor violation in the slepton mixing. Then, this slepton mixing is expected to contribute significantly to the $\mu \rightarrow e \gamma$ decay. The lepton-slepton interactions with the

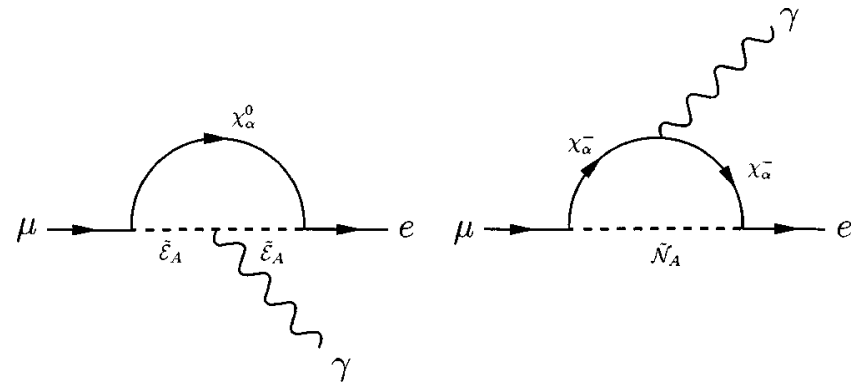

FIG. 1. One-loop diagrams for the $\mu \rightarrow e \gamma$ process mediated by the slepton mixing.

neutralinos $\chi_{\alpha}^{0}(\alpha=1-5)$ and the charginos $\chi_{\kappa}^{ \pm}(\kappa=1,2)$, which are relevant for $\mu \rightarrow e \gamma$, are given, respectively, by

$$
\mathcal{L}_{\chi^{0}}=N_{a \alpha A}^{R} \overline{\mathcal{E}}_{a} P_{R} \chi_{\alpha}^{0} \widetilde{\mathcal{E}}_{A}+N_{a \alpha A}^{L} \overline{\mathcal{E}}_{a} P_{L} \chi_{\alpha}^{0} \widetilde{\mathcal{E}}_{A}+\text { H.c. }
$$

and

$$
\mathcal{L}_{\chi^{ \pm}}=C_{a \kappa K}^{R} \overline{\mathcal{E}}_{a} P_{R} \chi_{\kappa}^{-} \tilde{\mathcal{N}}_{K}+C_{a \kappa K}^{L} \overline{\mathcal{E}}_{a} P_{L} \chi_{\kappa}^{-} \tilde{\mathcal{N}}_{K}+\text { H.c. },
$$

where $\widetilde{\mathcal{E}}_{A}(A=1-8)$ and $\tilde{\mathcal{N}}_{K}(K=1-5)$ are the slepton mass eigenstates. The coupling coefficients are given in terms of the transformation matrices to diagonalize the mass matrices of leptons, sleptons, charginos, and neutralinos.

We here evaluate the decay rate of $\mu \rightarrow e \gamma$ which is provided by the slepton mixing. By calculating the one-loop diagrams for the $\mu \longrightarrow e \gamma$ process mediated by the neutralinos and charginos, as shown in Fig. 1, we obtain the contributions to the decay amplitudes [7]

$$
\begin{aligned}
A_{R}^{(n)}= & \frac{1}{32 \pi^{2}} \sum_{A, \alpha}\left[\frac{m_{\mu}}{M_{\tilde{\mathcal{E}}_{A}}^{2}} N_{1 \alpha A}^{R} N_{2 \alpha A}^{R *} f_{1}\left(x_{\alpha A}\right)\right. \\
& \left.+\frac{M_{\chi_{\alpha}}^{0}}{M_{\tilde{\mathcal{E}}_{A}}^{2}} N_{1 \alpha A}^{R} N_{2 \alpha A}^{L *} f_{2}\left(x_{\alpha A}\right)\right], \\
A_{L}^{(n)}= & A_{R}^{(n)}(R \leftrightarrow L), \\
A_{R}^{(c)}= & \frac{1}{32 \pi^{2}} \sum_{K, \kappa}\left[\frac{m_{\mu}}{M_{\tilde{\mathcal{N}}_{K}}^{2}} C_{1 \kappa K}^{R} C_{2 \kappa K}^{R *} f_{3}\left(x_{\kappa K}\right)\right. \\
& \left.+\frac{M_{\chi_{\kappa}^{-}}}{M_{\tilde{\mathcal{N}}_{K}}^{2}} C_{1 \kappa K}^{R} C_{2 \kappa K}^{L *} f_{4}\left(x_{\kappa K}\right)\right], \\
A_{L}^{(c)}= & A_{R}^{(c)}(R \leftrightarrow L),
\end{aligned}
$$

where $f_{1}-f_{4}$ are certain functions of $x_{\alpha A} \equiv\left(M_{\chi_{\alpha}^{0}} / M_{\mathcal{E}_{A}}\right)^{2}$ and $x_{\kappa K} \equiv\left(M_{\chi_{\bar{\kappa}}} / M_{\tilde{\mathcal{N}}_{K}}\right)^{2}$. We estimate below $\operatorname{Br}(\mu \rightarrow e \gamma)$ with these contributions for the model (1) and (2). We will see that these models provide quite different results. In fact, these supersymmetric contributions are rather suppressed in the model (1) with the extra factor involving $m_{e}$ as well as $m_{\mu}$. 


\section{A. Model (1)}

The charged slepton mass matrix is given as

$$
\mathcal{M}_{\tilde{\mathcal{E}}}^{2}=\left(\begin{array}{cc}
M_{\widetilde{\mathcal{E}}_{L L}}^{2} & M_{\widetilde{\mathcal{E}}_{L R}}^{2} \\
M_{\widetilde{\mathcal{E}}_{R L}}^{2} & M_{\widetilde{\mathcal{E}}_{R R}}^{2}
\end{array}\right),
$$

where $L$ and $R$ represent the chirality of the corresponding leptons. The neutral slepton mass matrix is also given in a similar form. In order to see the flavor changing structure of the lepton-slepton-gaugino interactions, it is suitable to transform this slepton mass matrix by the orthogonal matrices which diagonalize the lepton mass matrix:

$$
\mathcal{M}_{\tilde{\mathcal{E}}}^{2^{\prime}}=\left(\begin{array}{cc}
U_{\mathcal{E}}^{T} & 0 \\
0 & V_{\mathcal{E}}^{T}
\end{array}\right) \mathcal{M}_{\tilde{\mathcal{E}}}^{2}\left(\begin{array}{cc}
U_{\mathcal{E}} & 0 \\
0 & V_{\mathcal{E}}
\end{array}\right)
$$

The universality for the soft supersymmetry breaking at $M_{G}$ is violated at $M_{W}$ due to the renormalization group effects. Then, the reduced slepton mass matrix $\mathcal{M}_{\tilde{\mathcal{E}}}^{2^{\prime}}$ involves the flavor changing elements.

In the model (1), the components of the slepton mass matrix are given including the renormalization group effects as

$$
\begin{aligned}
& M_{\widetilde{\mathcal{E}}_{L L}}^{2}=x_{L L} M_{\mathcal{E}} M_{\mathcal{E}}^{T}+y_{L L} m_{0}^{2} \mathbf{1}_{4} \\
& +m_{0}^{2}\left(\begin{array}{cc}
c_{L L}^{i j}\left(\lambda_{1}^{i i}\right)^{2}+c_{L L}^{j i}\left(\lambda_{1}^{j j}\right)^{2} & d_{L L}^{i}\left(\lambda_{1}^{i i}\right)^{2} \\
d_{L L}^{j}\left(\lambda_{1}^{j j}\right)^{2} & 0
\end{array}\right), \\
& M_{\widetilde{\mathcal{E}}_{L R}}^{2}=\left(M_{\widetilde{\mathcal{E}}_{R L}}^{2}\right)^{T}=x_{L R} m_{0} M_{\mathcal{E}}+x_{L R}^{\prime} m_{0}\left(\mathbf{0} \quad M_{\mathcal{N}}\right) \\
& +m_{0}^{2}\left(\begin{array}{cc}
\delta_{i j} f_{L R}^{i} \lambda_{1}^{i i} & g_{L R}^{i}\left(\lambda_{1}^{i i}\right)^{2} \\
\mathbf{0} & 0
\end{array}\right), \\
& M_{\tilde{\mathcal{E}}_{R R}}^{2}=x_{R R} M_{\mathcal{E}}^{T} M_{\mathcal{E}}+y_{R R} m_{0}^{2} \mathbf{1}_{4}+z_{R R} m_{0}^{2}\left(\begin{array}{ll}
\mathbf{0} & \mathbf{0} \\
\mathbf{0} & 1
\end{array}\right) \\
& +m_{0}^{2}\left(\begin{array}{cc}
\delta_{i j} c_{R R}^{i}\left(\lambda_{1}^{i i}\right)^{2} & \mathbf{0} \\
\mathbf{0} & 0
\end{array}\right)
\end{aligned}
$$

where $x_{L L}, \ldots \sim 1$ are the relevant parameters. The terms proportional to the $4 \times 4$ unit matrix $\mathbf{1}_{4}$ as well as those given by the charged lepton mass matrix $M_{\mathcal{E}}$ are diagonalized by the transformation in Eq. (39). The term given by the neutral lepton mass matrix $M_{\mathcal{N}}$ is almost diagonalized by the similarity of $U_{\mathcal{E}}^{T} O_{\mathcal{N}}$. The third term of Eq. (42) provides a contribution $\left(V_{\mathcal{E}}\right)_{4 i}\left(V_{\mathcal{E}}\right)_{4 j}$ for the slepton mixing, which is substantially suppressed by the lepton mass ratios $\left(m_{e_{i}} /\right.$ $\left.M_{E}\right)\left(m_{e_{j}} / M_{E}\right)$. The remaining terms involve the Yukawa couplings $\lambda_{1}^{i i} \sim m_{e_{i}} / v_{1}$. Among these contributions in the model (1), the significant flavor mixing arises from the fourth term of Eq. (42) in the right-handed charged slepton sector as

$$
\left(M_{\tilde{\mathcal{E}}_{R R}}^{2^{\prime}}\right)_{i j} / m_{0}^{2} \sim\left(V_{\mathcal{E}}^{T}\right)_{i i}\left(\lambda_{1}^{i i}\right)^{2}\left(V_{\mathcal{E}}\right)_{i j}+\left(V_{\mathcal{E}}^{T}\right)_{i j}\left(\lambda_{1}^{j j}\right)^{2}\left(V_{\mathcal{E}}\right)_{j j}
$$

It is here noted that the right-handed ordinary charged lepton mixing induced by the ordinary-exotic lepton mixing is related to the charged lepton masses as

$$
\left(V_{\mathcal{E}}\right)_{i j} \sim \frac{m_{e_{i}} m_{e_{j}}}{m_{e_{i}}^{2}+m_{e_{j}}^{2}} \lambda_{2}^{i 4} \lambda_{2}^{j 4}(i \neq j) .
$$

By considering these arguments on the slepton mixing, the dominant contribution from the neutralino couplings to the decay amplitude of $\mu \rightarrow e \gamma$ is estimated as

$$
A_{L}^{(n)} \sim \frac{1}{32 \pi^{2}} \frac{\lambda_{3} \lambda_{1}^{22} v_{2} v_{3}}{m_{0}^{3}}\left(\lambda_{1}^{22}\right)^{2}\left(m_{e} / m_{\mu}\right) \lambda_{2}^{14} \lambda_{2}^{24} .
$$

Here, the right-handed $\widetilde{\mu}^{c}-\widetilde{e}^{c}$ slepton mixing $\sim\left(\lambda_{1}^{22}\right)^{2}\left(V_{\mathcal{E}}\right)_{12}$ given by Eqs. (43) and (44) is used for the $e_{R^{-}}-\tilde{\mu}^{c}-\chi^{0}$ vertex, and the chirality flip $\sim \lambda_{3} \lambda_{1}^{22} v_{2} v_{3}$ of the intermediate $\tilde{\mu}$ state is provided by the $\left|F_{H_{1}}\right|^{2}$ term. The chargino couplings provide a contribution of the same order as Eq. (45), where the chirality flipping $\widetilde{N}-\widetilde{e}^{c}$ slepton mixing given by $\left(O_{\mathcal{E}}^{T} M_{\tilde{\mathcal{N}}_{L R}}^{2}\right)_{41} / m_{0} \sim\left(m_{e} / m_{0}\right) \tan \beta \lambda_{2}^{14}$ is used for the $e_{R}-\widetilde{N}-\chi_{0}$ vertex, and the left-handed $\widetilde{N}-\widetilde{\nu}_{\mu}$ slepton mixing in the intermediate state is provided by $\left(O_{\mathcal{E}}^{T} M_{\tilde{\mathcal{N}}_{L L}}^{2}\right)_{42} / m_{0}^{2}$ $\sim\left(m_{\mu} / M_{W}\right)^{2} \tan \beta \lambda_{2}^{24}$. Accordingly, in the model (1) the contributions to the decay amplitude are dominantly given by

$$
A_{L}^{(n)} \sim A_{L}^{(c)} \sim \frac{1}{32 \pi^{2}} \tan ^{3} \beta \frac{m_{e} m_{\mu}^{2}}{M_{W}^{2} m_{0}^{2}} \lambda_{2}^{14} \lambda_{2}^{24},
$$

where $v_{3} \sim m_{0}$ is considered for definiteness. These contributions should be compared to the nonsupersymmetric contributions given in Eq. (24). Although these supersymmetric contributions have the specific dependence on the relevant lepton masses similar to Eq. (24), it can be enhanced significantly by the power of $\tan \beta$. We roughly estimate the branching ratio as

$$
\begin{aligned}
\operatorname{Br}(\mu \rightarrow e \gamma) & \sim \frac{3 \alpha}{32 \pi} \tan ^{6} \beta\left(\frac{m_{e} m_{\mu}}{m_{0}^{2}}\right)^{2}\left(\lambda_{2}^{14} \lambda_{2}^{24}\right)^{2} \\
& \sim 10^{-13}\left(\tan \beta \sim 20, \lambda_{2}^{14} \sim \lambda_{2}^{24} \sim 0.3\right) .
\end{aligned}
$$

It should here be remarked as seen in Eq. (46) that in the model (1) the chirality of the charged leptons is mainly specified as $\mu_{L}^{-} \rightarrow e_{R}^{-} \gamma$.

The dependence of the decay amplitudes on both $m_{e}$ and $m_{\mu}$, as seen in Eq. (46), can be understood as follows by means of approximate flavor symmetries. Set $\lambda_{1}^{11}\left(M_{G}\right)=0$ in Eq. (3). Then, we can make $\lambda_{2}^{14}\left(M_{G}\right)=0$ by a suitable transformation of the left-handed lepton doublets $l_{1}$ and $l_{4}=L$, while keeping $\lambda_{1}\left(M_{G}\right)$ with $\lambda_{1}^{11}\left(M_{G}\right)=0$. In this limit, a flavor symmetry $\mathrm{U}(1)_{e}$ appears under the phase transforma- 


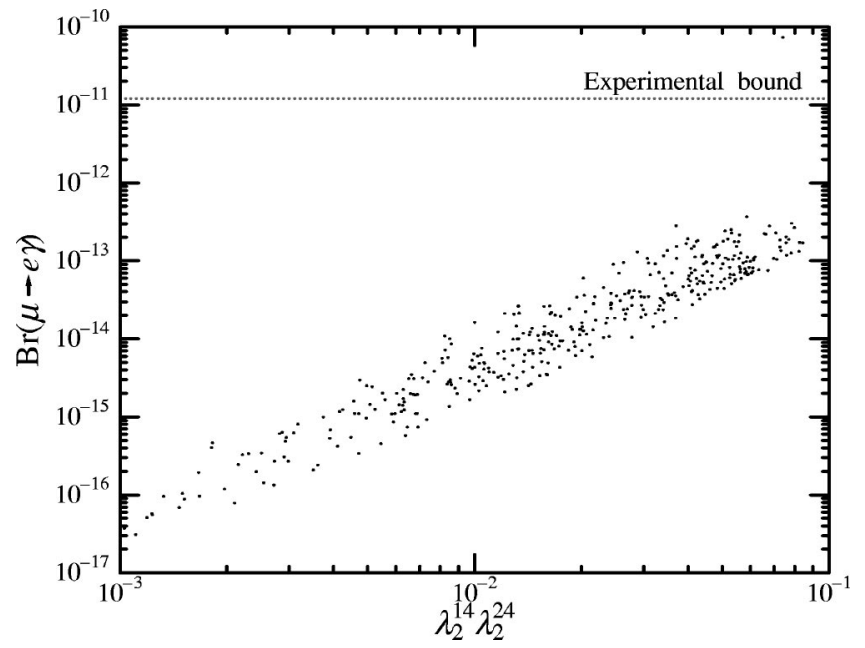

FIG. 2. The relation between $\operatorname{Br}(\mu \rightarrow e \gamma)$ and $\lambda_{2}^{14} \lambda_{2}^{24}$ is plotted in the model (1) for $\tan \beta=20$. The points represent the branching ratio for random values of $\lambda_{2}^{i 4}$.

tion $l_{1} \rightarrow e^{i \alpha} l_{1}$ and $e_{1}^{c} \rightarrow e^{-i \alpha} e_{1}^{c}$. If the universal form is assumed for the supersymmetry breaking terms at $M_{G}$, this flavor symmetry $\mathrm{U}(1)_{e}$ is still preserved in the limit $\lambda_{1}^{11}\left(M_{G}\right)=0$. This is the case even if the renormalization group corrections are included. Therefore, we find that in the limit $\lambda_{1}^{11}\left(M_{G}\right)=0$ corresponding to $m_{e}=0$, the $\mu \rightarrow e \gamma$ decay is prevented for the electron number conservation by the $\mathrm{U}(1)_{e}$. Similarly, the decay amplitudes of $\mu \rightarrow e \gamma$ depend on $m_{\mu}$ as well due to the approximate $\mathrm{U}(1)_{\mu}$ symmetry. It should further be remarked that even for the case with several pairs of vectorlike lepton doublets, the $\mu \rightarrow e \gamma$ decay amplitudes are dominantly given by Eq. (46) in the model (1) without the bare mass term $M_{L} L L^{c}$. This can be justified by extending readily the above symmetry argument.

In order to confirm the estimate of $\operatorname{Br}(\mu \rightarrow e \gamma)$ given in Eq. (47), we have made numerical calculations by taking reasonable values of the model parameters. In the model (1), the most important parameters are $\tan \beta$ and $\lambda_{2}^{i 4}$ for the ordinary-exotic lepton mixing. The values of $\lambda_{1}^{i i}$ are determined so as to reproduce the actual lepton masses $m_{e}, m_{\mu}, m_{\tau}$. The exotic leptons $E$ and $N$ have typically the masses $M_{E} \simeq M_{N} \sim 100 \mathrm{GeV}$ by taking $\lambda_{2}^{44} \sim 0.3$ and $v_{3}$ $\sim 500 \mathrm{GeV}$.

In Fig. $2, \operatorname{Br}(\mu \rightarrow e \gamma)$ is shown as a function of $\lambda_{2}^{14} \lambda_{2}^{24}$ by taking randomly the values of $\lambda_{2}^{i 4}$. We have also taken typically $\tan \beta=20, v_{3}=500 \mathrm{GeV}, m_{0}=100 \mathrm{GeV}, a_{0}=1, m_{1}$ $=100 \mathrm{GeV}, \lambda_{2}^{44}=0.3, \lambda_{3}=0.6$, and $\lambda_{4}=0.5$. It is clearly observed in Fig. 2 that $\operatorname{Br}(\mu \rightarrow e \gamma)$ is roughly proportional to $\left(\lambda_{2}^{14} \lambda_{2}^{24}\right)^{2}$, as indicated in Eq. (47).

The relation between $\operatorname{Br}(\mu \rightarrow e \gamma)$ and $\tan \beta$ is shown in Fig. 3. Here, we have taken typically $v_{3}=500 \mathrm{GeV}, m_{0}$ $=200 \mathrm{GeV}, a_{0}=0, m_{1}=200 \mathrm{GeV}, \lambda_{2}^{a 4}=0.3, \lambda_{3}=0.6$, and $\lambda_{4}=0.5$. This result indicates the strong $\tan \beta$ dependence as $\operatorname{Br}(\mu \rightarrow e \gamma) \propto \tan ^{6} \beta$. It is, in particular, interesting that if $\tan \beta$ is larger than $20, \operatorname{Br}(\mu \rightarrow e \gamma)$ might be comparable to the experimental bound $1.2 \times 10^{-11}$ [14].

As for the $\mu \rightarrow 3 e$ decay, the penguin diagrams associated

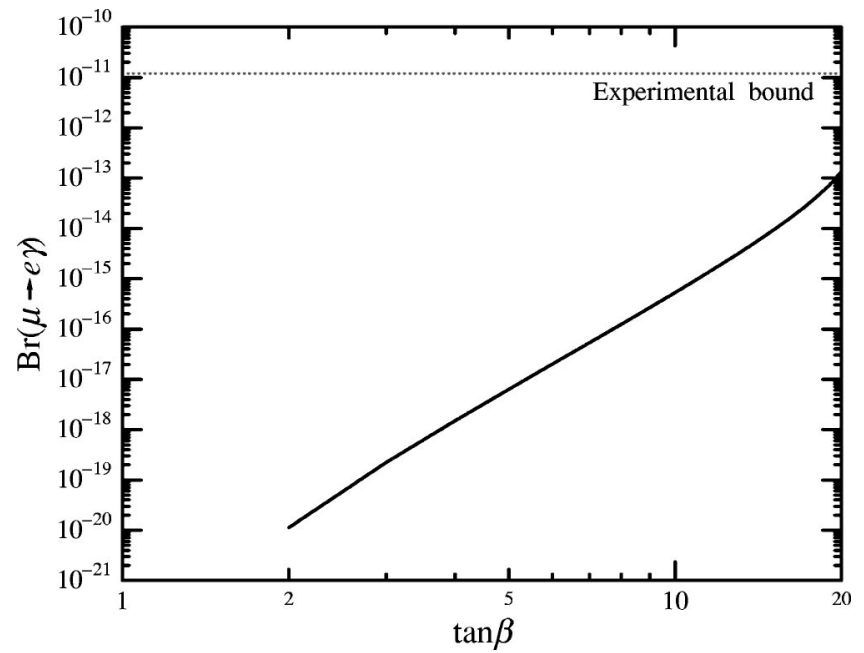

FIG. 3. The relation between $\operatorname{Br}(\mu \rightarrow e \gamma)$ and $\tan \beta$ is plotted in the model (1) for $\lambda_{2}^{a 4}=0.3$.

with Fig. 1 provide dominant contributions in the present model with $\operatorname{Br}(\mu \rightarrow 3 e) / \operatorname{Br}(\mu \rightarrow e \gamma) \sim 7 \times 10^{-3}$, as is usually the case [7]. The tree-level $Z$ mediated FCNC's given in Eq. (21) provide only a contribution smaller by a few orders. We have also the supersymmetric contributions to $\tau \rightarrow e \gamma$ and $\tau \longrightarrow \mu \gamma$, which are estimated in Eq. (47) by replacing the relevant charged lepton masses and the $\lambda_{2}^{i 4}$ couplings. In particular, $\operatorname{Br}(\tau \rightarrow \mu \gamma)$ is enhanced as

$$
\begin{aligned}
\operatorname{Br}(\tau \rightarrow \mu \gamma) & \sim\left(\frac{m_{\mu} m_{\tau}}{m_{e} m_{\mu}}\right)^{2} \operatorname{Br}(\mu \rightarrow e \gamma) \\
& \sim 10^{-7}\left(\tan \beta \sim 20, \lambda_{2}^{24} \sim \lambda_{2}^{34} \sim 0.3\right),
\end{aligned}
$$

which can be comparable to the experimental bound $1.1 \times 10^{-6}[15]$. Therefore, in the model (1) the $\tau \rightarrow \mu \gamma$ decay seems to be more promising to observe the lepton flavor violation due to the ordinary-exotic lepton mixing with supersymmetry.

The $\lambda_{2}$ coupling may also contribute to the flavor violation in the quark sector if the quark singlets $\left(D^{c}, D\right)$ survive as well to the electroweak scale in the $E_{6}$ unification. Then, we have dominantly the right-handed squark mixings such as

$$
\begin{aligned}
\left(M_{\tilde{\mathcal{D}}_{R R}}^{2 \prime}\right)_{12} / m_{0}^{2} & \sim\left(m_{s} m_{d} / v^{2}\right) \lambda_{2}^{14} \lambda_{2}^{24} \tan ^{2} \beta \\
& \sim 10^{-8} \lambda_{2}^{14} \lambda_{2}^{24} \tan ^{2} \beta,
\end{aligned}
$$

which are the same as the right-handed slepton mixings given in Eqs. (43) with (44). It is clearly found that even for $\tan \beta \sim 50$ this squark mixing is much smaller than the bound $\sim 10^{-2}$ obtained from the $K^{0}-\bar{K}^{0}$ system [16].

\section{B. Model (2)}

In the model (2), we have more significant contributions for the right-handed decay amplitudes. This is because the $\mathrm{U}(1)_{e} \times \mathrm{U}(1)_{\mu}$ considered in the case of model (1) is substantially violated due to the presence of bare mass term $M_{L} L L^{c}$ 
of the vectorlike lepton doublets. Actually, the soft supersymmetry breaking mass terms for the ordinary slepton doublets $\widetilde{l}$ and the vectorlike slepton doublet $\widetilde{L}$ acquire the renormalization group corrections in somewhat different way with $\lambda_{2}^{44} \equiv 0$. Then, we have an extra term for $M_{\tilde{\mathcal{E}}_{L L}}^{2}$ and $M_{\tilde{\mathcal{N}}_{L L}}^{2}$ as

$$
z_{L L} m_{0}^{2}\left(\begin{array}{cc}
\mathbf{0} & \mathbf{0} \\
\mathbf{0} & 1
\end{array}\right) \rightarrow M_{\widetilde{\mathcal{E}}_{L L}}^{2}, M_{\widetilde{\mathcal{N}}_{L L}}^{2}
$$

This extra term provides the significant mixing for the lefthanded sleptons as

$$
\left(M_{\tilde{\mathcal{E}}_{L L}}^{2 \prime}\right)_{i j} / m_{0}^{2} \sim\left(M_{\tilde{\mathcal{N}}_{L L}}^{2 \prime}\right)_{i j} / m_{0}^{2} \sim\left(U_{\mathcal{E}}^{T}\right)_{i 4}\left(U_{\mathcal{E}}\right)_{4 j} \sim \lambda_{2}^{i 4} \lambda_{2}^{j 4},
$$

where the similarity $U_{\mathcal{E}} \simeq O_{\mathcal{N}}$ is considered. These lefthanded slepton mixings can be taken for the one-loop diagrams similar to those considered in the model (1) with chirality change $L \leftrightarrow R$. Then, we obtain the contributions to the $\mu \rightarrow e \gamma$ decay amplitude as

$$
A_{R}^{(n)} \sim A_{R}^{(c)} \sim \frac{1}{32 \pi^{2}} \tan \beta \frac{m_{\mu}}{m_{0}^{2}} \lambda_{2}^{14} \lambda_{2}^{24} .
$$

It is here important that these supersymmetric contributions are no longer suppressed by the very small extra factor $m_{e} m_{\mu} / M_{W}^{2}$ as seen in Eq. (46) for the model (1). We roughly estimate the branching ratio as

$$
\begin{aligned}
\operatorname{Br}(\mu \rightarrow e \gamma) & \sim \frac{3 \alpha}{32 \pi} \tan ^{2} \beta\left(\frac{M_{W}}{m_{0}}\right)^{4}\left(\lambda_{2}^{14} \lambda_{2}^{24}\right)^{2} \\
& \sim 10^{-11}\left(\tan \beta \sim 1, \lambda_{2}^{14} \sim \lambda_{2}^{24} \sim 0.01\right) .
\end{aligned}
$$

As seen in Eq. (51), the chirality of the charged leptons is mainly specified as $\mu_{R}^{-} \rightarrow e_{L}^{-} \gamma$ in the model (2) contrary to the case of model (1). It is also mentioned that this significant supersymmetric contribution to $\operatorname{Br}(\mu \rightarrow e \gamma)$ may be obtained even in the model (1), if the soft slepton masses squared $m_{\tilde{l}}^{2}$ are different between $\widetilde{l}_{i}$ and $\widetilde{l}_{4}=\widetilde{L}$ already at $M_{G}$ for some reason.

We have also made numerical calculations to evaluate $\operatorname{Br}(\mu \rightarrow e \gamma)$ in the model (2) with the bare mass term, which is expected to be much larger than in the model (1). In Fig. 4, $\operatorname{Br}(\mu \rightarrow e \gamma)$ is shown as a function of $\lambda_{2}^{14} \lambda_{2}^{24}$ by taking randomly the values of $\lambda_{2}^{i 4}$. We have also taken typically $\tan \beta=5, \quad v_{3}=500 \mathrm{GeV}, \quad m_{0}=300 \mathrm{GeV}, \quad a_{0}=1, \quad m_{1}$ $=300 \mathrm{GeV}, M_{L}=100 \mathrm{GeV}, \lambda_{3}=0.8$, and $\lambda_{4}=0.7$. It is clearly observed in Fig. 4 that $\operatorname{Br}(\mu \rightarrow e \gamma)$ is roughly proportional to $\left(\lambda_{2}^{14} \lambda_{2}^{24}\right)^{2}$, as indicated in Eq. (52). It is interesting to observe in Fig. 4 that $\operatorname{Br}(\mu \rightarrow e \gamma)$ becomes comparable to the experimental bound $1.2 \times 10^{-11}$ [14] for the rather small ordinary-exotic couplings $\left(\lambda_{2}^{14} \lambda_{2}^{24}\right)^{1 / 2} \sim 0.02$ with the moderate value of $\tan \beta \sim 5$.

The relation between $\operatorname{Br}(\mu \rightarrow e \gamma)$ and the gravitino mass $m_{0}$ is shown in Fig. 5 for $m_{0} \leqslant 3 \mathrm{TeV}$ with somewhat larger

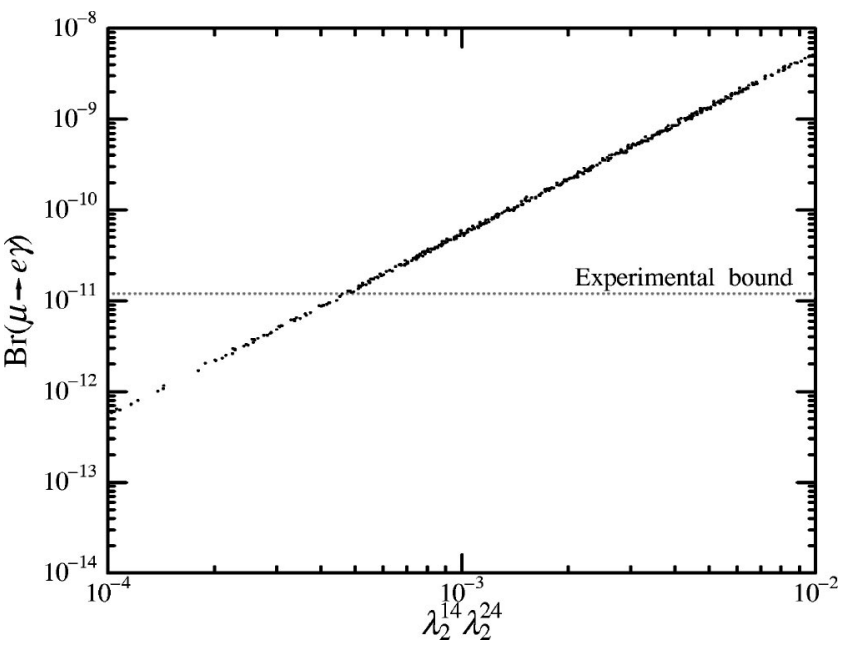

FIG. 4. The relation between $\operatorname{Br}(\mu \rightarrow e \gamma)$ and $\lambda_{2}^{14} \lambda_{2}^{24}$ is plotted in the model (2) for $\tan \beta=5$. The points represent the branching ratio for random values of $\lambda_{2}^{i 4}$.

$\lambda_{2}^{i 4}=0.07$, where the other parameters are taken typically as $\tan \beta=5, \quad v_{3}=500 \mathrm{GeV}, \quad a_{0}=1, \quad m_{1}=1000 \mathrm{GeV}, \quad M_{L}$ $=100 \mathrm{GeV}, \lambda_{3}=0.8$, and $\lambda_{4}=0.7$. It is here noticed that cancellation between the neutralino and chargino contributions occurs for certain values of $m_{0}$, where $\operatorname{Br}(\mu \rightarrow e \gamma)$ becomes substantially small. It is also remarkable in the model (2) that even for the relatively large soft supersymmetry breaking with $m_{0} \sim 1 \mathrm{TeV}, \operatorname{Br}(\mu \rightarrow e \gamma)$ can be comparable to the experimental bound by taking the relatively large ordinary-exotic lepton couplings $\lambda_{2}^{i 4} \sim 0.1$.

We have also the supersymmetric contributions to $\tau$ $\rightarrow e \gamma$ and $\tau \rightarrow \mu \gamma$, which are estimated in Eq. (51) by replacing $m_{\mu}$ with $m_{\tau}$ and the $\lambda_{2}^{i 4}$ couplings. The resultant branching ratios of $\tau \rightarrow e \gamma$ and $\tau \rightarrow \mu \gamma$ are similar to that of $\mu$ $\rightarrow e \gamma$, since they are almost independent of $m_{\tau}$ in this case. Therefore, by considering the experimental bounds on the branching ratios, the $\mu \rightarrow e \gamma$ seems to be more promising to

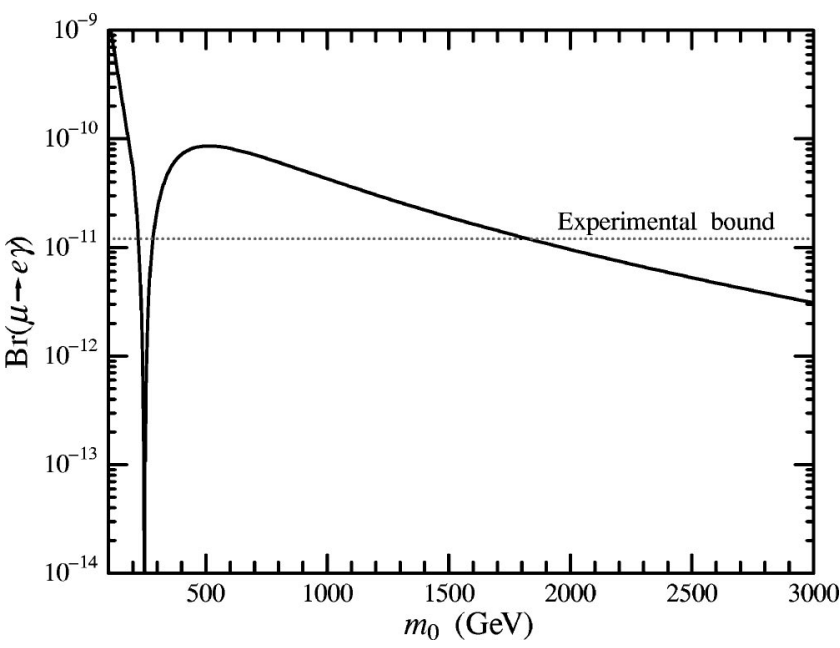

FIG. 5. The relation between $\operatorname{Br}(\mu \rightarrow e \gamma)$ and the gravitino mass $m_{0}$ is plotted in the model (2) for $m_{0} \leqslant 3 \mathrm{TeV}$ with $\lambda_{2}^{i 4}=0.07$ and $\tan \beta=5$. 
observe the lepton flavor violation in the model (2) with the bare mass term, if the ordinary-exotic lepton couplings $\lambda_{2}^{i 4}$ are comparable to each other.

The possible contributions of the $\lambda_{2}$ coupling to the flavor violation in the quark sector are estimated in this model (2) as follows. We have here the significant left-handed squark mixings such as $\left(M_{\tilde{\mathcal{D}}_{L L}}^{2 \prime}\right)_{12} / m_{0}^{2} \sim \lambda_{2}^{14} \lambda_{2}^{24}$, which is the same as the left-handed slepton mixings given in Eq. (50). Then, a constraint $\lambda_{2}^{14} \lambda_{2}^{24} \lesssim 10^{-2}$ is placed from the $K^{0}-\bar{K}^{0}$ system [16]. This constraint is, however, less stringent than that from the $\mu \rightarrow e \gamma$.

\section{SUMMARY}

In summary, we have investigated the lepton flavor violating processes, especially the $\mu \rightarrow e \gamma$ decay, which are obtained from the mixing between the ordinary leptons and the vectorlike leptons. Although the lepton FCNC's appear at the tree level, their effects are small naturally because of the hierarchy of the charged lepton masses. Hence, the fine tuning of parameters is not necessary to suppress the FCNC's sufficiently. In the supersymmetric model, significant effects of lepton flavor violation are obtained from the slepton mixing which is generated by radiative corrections with the ordinary-exotic lepton couplings. In the model (1) without the bare mass term of the vectorlike lepton doublets, the supersymmetric contributions to the $\mu \rightarrow e \gamma$ decay are rather suppressed by $m_{e}$ as well as $m_{\mu}$ due to the approximate $\mathrm{U}(1)_{e} \times \mathrm{U}(1)_{\mu}$ symmetry, which is similar to the gauge boson mediated contributions. It is, however, remarkable that these supersymmetric contributions to $\operatorname{Br}(\mu \rightarrow e \gamma)$ are substantially enhanced by the factor $\tan ^{6} \beta$ compared to the gauge boson mediated contributions. Then, if $\tan \beta$ is larger than
$20, \operatorname{Br}(\mu \rightarrow e \gamma)$ might be comparable to the present experimental bound. In the model (2) with the bare mass term, much larger contributions to $\operatorname{Br}(\mu \rightarrow e \gamma)$ are obtained through the slepton mixing. In either case we have shown that the slepton mixing contributions to the $\mu \rightarrow e \gamma$ decay can be large enough to be observed in the near future experiments. It should also be noted that in the model (1) the $\tau$ $\rightarrow \mu \gamma$ decay may be more promising as seen in Eq. (48).

The discovery of $\mu \rightarrow e \gamma$ clearly indicates the new physics. The supersymmetric contributions to the $\mu \rightarrow e \gamma$ decay have been considered so far in the literature for other supersymmetric models such as the SUSY GUT [3-5] and the MSSM with right-handed neutrinos [6-8]. The predictions of $\operatorname{Br}(\mu \rightarrow e \gamma)$ in these models are within reach of the future experiments. These are, however, the effects of ultra high energy physics much above the electroweak scale. In the supersymmetric model with vectorlike leptons, which may be motivated by the $E_{6}$ type unification, the significant contributions to the $\mu \rightarrow e \gamma$ decay can be obtained due to the exotic leptons and their scalar partners which exist just around the electroweak scale. Therefore, the direct experimental search is feasible for these new particles $[12,17]$. It is also observed in the model (2) with the bare mass term of vectorlike leptons that even if the soft supersymmetry breaking scale is around $1 \mathrm{TeV}, \operatorname{Br}(\mu \rightarrow e \gamma)$ can be comparable to the current experimental bound with relatively large ordinary-exotic lepton couplings. These features are salient to the supersymmetric model with vectorlike leptons in contrast to the other supersymmetric models.

\section{ACKNOWLEDGMENTS}

We would like to thank Y. Okada and J. Hisano for useful discussions.
[1] For a review of phenomenological aspects of the stringinspired models, see for instance, J. L. Hewett and T. G. Rizzo, Phys. Rep. 183, 193 (1989).

[2] A. H. Chamseddine, R. Arnowitt, and P. Nath, Phys. Rev. Lett. 49, 970 (1982); R. Barbieri, S. Ferrara, and C. A. Savoy, Phys. Lett. 119B, 343 (1982); L. Hall, J. Lykken, and S. Weinberg, Phys. Rev. D 27, 2359 (1983).

[3] R. Barbieri and L. J. Hall, Phys. Lett. B 338, 212 (1994).

[4] R. Barbieri, L. Hall, and A. Strumia, Nucl. Phys. B445, 219 (1995).

[5] J. Hisano, D. Nomura, Y. Okada, Y. Shimizu, and M. Tanaka, Phys. Rev. D 58, 116010 (1998).

[6] F. Borzumati and A. Masiero, Phys. Rev. Lett. 57, 961 (1986).

[7] J. Hisano, T. Moroi, K. Tobe, and M. Yamaguchi, Phys. Rev. D 53, 2442 (1996).

[8] J. Hisano and D. Nomura, Phys. Rev. D 59, 116005 (1999).

[9] P. Langacker and D. London, Phys. Rev. D 38, 907 (1988).

[10] S. L. Dubovsky and D. S. Gorbunov, Phys. Lett. B 419, 223 (1998); Nucl. Phys. B557, 119 (1999).
[11] T. Yanagida, in Proceedings of the Workshop on Unified Theory and Baryon Number of the Universe, edited by $\mathrm{O}$. Sawada and A. Sugamoto (KEK, Tokyo, 1975), p. 95; M. Gell-Mann, P. Ramond, and R. Slansky, in Supergravity, edited by P. van Nieuwenhuizen and D. Freedman (North Holland, Amsterdam, 1979).

[12] L3 Collaboration, L3 report, LEPC, 1999.

[13] L. J. Hall, V. A. Kostelecky, and S. Raby, Nucl. Phys. B267, 415 (1986).

[14] MEGA Collaboration, M. L. Brooks et al., Phys. Rev. Lett. 83, 1521 (1999).

[15] CLEO Collaboration, S. Ahmed et al., Phys. Rev. D 61, 071101 (2000).

[16] F. Gabbiani, E. Gabrielli, A. Masiero, and L. Silvestrini, Nucl. Phys. B477, 321 (1996).

[17] Y. A. Coutinho, A. J. Ramalho, and S. Wulck, Phys. Rev. D 54, 1215 (1996); F. M. L. Almeida et al., ibid. 51, 5990 (1995). 